Classification

Physics Abstracts

06.60.Mr - 81.70.Fy

\title{
Quantification of Orientation of Pore Patterns in X-ray Images of Deformed Clay
}

\author{
Alain Pierret $\left({ }^{1,2}\right)$ and Christopher J. Moran $\left({ }^{2}\right)$ \\ $\left({ }^{1}\right)$ Laboratoire de Physique et Biologie des Sols, INRA Zoologie, Domaine St-Paul, Site Agroparc, \\ 84914 Avignon Cedex 9, France \\ $\left({ }^{2}\right)$ Commonwealth Scientific and Industrial Research Organization, Division of Soils, GPO Box 639, \\ ACT 2601, Australia
}

\begin{abstract}
Résumé. - Les techniques radiographiques sont couramment employées dans le cadre de l'analyse technologique des céramiques archéologiques, et permettent d'estimer la variabilité des techniques de fabrication. Dans cet article, une méthode de mesure de l'orientation des pores est présentée. Cette méthode est appliquée à des céramiques d'origine connue dont la structure est significative du régime de déformation mis en jeu lors du façonnage.
\end{abstract}

\begin{abstract}
The use of X-ray imaging to assess variability in ceramic fabrication is common in archaeological studies aimed at examining ancient pottery technologies. In this paper, a method based on the measurement of individual pores orientation is presented. This method is successfully applied to ceramic specimens of known origin whose structure signified different deformation histories.
\end{abstract}

\section{Introduction}

In many fields of investigation, e.g. medical checkups, non-destructive material analysis, mineral or metallurgical content analysis, X-ray imaging techniques have been demonstrated to provide valuable qualitative data on the internal structure of most of the living creatures and materials. The use of X-ray imaging to assess variability in ceramic fabrication is common in archaeological studies aimed at examining ancient pottery technologies [1-7]. These studies are based on empirical observations that the various deformation rates applied to clay for forming vessels according to different techniques, induce specific structural patterns that may be detected visually on X-radiographs. Key indicators include orientation of particles and voids and shapes of voids. One presents in this paper that it is possible to go beyond this simple qualitative description and to assess whether or not the pore space presents a preferred orientation. For this purpose, one considers successively the results from two methods: 1) the grey-level granulometry using isotropic and anisotropic structuring elements, and 2) a method based on the measurement of orientation of individual pores. One demonstrates that, contrary to the grey-level granulometry, this later 
method allows to separate so bent from straight porous patterns, and is subsequently well suited to the technological examination of ceramic specimens.

\section{Porosity Image Acquisition and Derivation}

The digital X-ray images used were obtained following a two step procedure. In the first step, radiographic exposures of the samples were obtained on Kodak Industrex $\mathbf{R}$ film. The resulting image depends on the X-ray intensity received after attenuation by the sample. X-ray attenuation through a homogeneous substance is given by Lambert's law and is proportional to sample thickness, gravimetric density and the attenuation coefficient. To reduce geometrical distortions, samples are imaged as close as possible to the X-ray beam axis and the emulsion surface [9]. Thickness of samples should not exceed $10 \mathrm{~mm}$ in order to limit image distortion related to the integration of three-dimensional information onto a plane.

The second step of the procedure is to digitize the radiographs using a document-scanner. Images were scanned at a resolution of $300 \mathrm{dpi}$, which results in a pixel length and width of $84 \mu \mathrm{m}$. To perform correct thickness and porosity assessments from Lambert's law, it was necessary to know the relation between the image grey-levels and the transmitted X-ray intensities. An empirical calibration was conducted using a wedge of quartzite 0 to $10 \mathrm{~mm}$ thick for some reference exposures. It was found that the film/scanner response is quite linear within a grey-level range from 30 to 255 .

Pierret et al. [8] showed how Lambert's Law of X-ray attenuation combined with some simple measurements can be used to separate thickness variation from porosity information in radiographs of any ceramic fragment. This results in a calibrated porosity image which is used to quantify the orientation of pore space.

\section{Orientation Quantification}

3.1 Grey-Level Method (FIXed GRID). - The pore patterns visible in porosity images are complex structures because of the geometrical projection associated with the radiographic technique. With this mode of image acquisition, superposition of individual components is unavoidable. Despite the structural complexity of these images, their physical significance is rather simple: one can consider the resulting porosity image as the map of the gravimetric density anomalies present throughout the sample.

Given the nature of the data, it was desirable to attempt to characterize the orientation pattern using a technique applicable to the continuous porosity phase $[0,1]$. An apparently suitable technique is the grey level granulometry [10] using linear structuring elements with a range of orientations. With an isotropic structuring element, the sequence of morphological closings (dilations followed by erosions) characterize the proportion of the total porosity accounted for by each successive size (where the size is the length of the structuring element).

With anisotropic structuring elements, e.g. linear, one can compare the amount of porosity versus size in different directions. Directions clockwise from the horizontal of $0^{\circ}, 45^{\circ}, 90^{\circ}$ and $135^{\circ}$ are derived from the eight neighbours of any given pixel. Additional angles of $60^{\circ}$ and $120^{\circ}$ are given using a hexagonal grid approximation.

The method is illustrated using two test images: one with straight oblique anisotropy at $135^{\circ}$ to the horizontal (Fig. 1a) and the other with bent anisotropy (Fig. 1b). The plots of proportion of porosity versus length of the element (or iteration number) are interpreted by examining the position of peaks. Peaks generally correspond to pores of a given dimension in a given direction. The existence of a peak indicates the presence of a class of porous components which size is given by the corresponding iteration number. 
For the case of straight anisotropy (Figs. 1c and 1d), the extent of porous components in the $0^{\circ}, 60^{\circ}, 90^{\circ}$ and $120^{\circ}$ directions are not distinguishable, i.e. all have peaks around 20 iterations. However, the alignment of most of the major axes at $135^{\circ}$ becomes clear when peaks are compared with the $45^{\circ}$ direction. Therefore, this straight anisotropy is detected and qualified using the grey level granulometry. For the bent pattern, there are no apparent complementary peaks in any two orthogonal directions (Fig. 1e). In fact, clear peaks are only evident at $45^{\circ}$. This is insufficient evidence of anisotropy. It is possible that we could attempt to remove the bending in the pattern prior to quantification of anisotropy by applying a warping operation to the image. Unfortunately, the arbitrary nature of selection of control points would render the derived characterization very difficult to interpret. Therefore, for bent anisotropy, other methods are required. One such method is described below.

\subsection{Binary Method.}

3.2.1 Image Segmentation. - The purpose of segmentation is to select individual pores, i.e., allocate each pixel to either the solid or the pore phase. The grey-level histograms of the porosity images show one dominant peak centered about the mean porosity value, while other values are spread following a distribution which loosely approximates normal distribution (Fig. 2).

There is a sharply-defined gap between the mean porosity value peak and larger porosity values. Therefore, the porosity images were segmented by threshold from the value corresponding to the first peak just beside the mean porosity value peak. Practically, this corresponds to a cutoff value of $0.99 \times$ the average porosity of each sample. This implies that only large pores or groups of small pores are selected. The components selected after segmentation are a particular representation of individual pores.

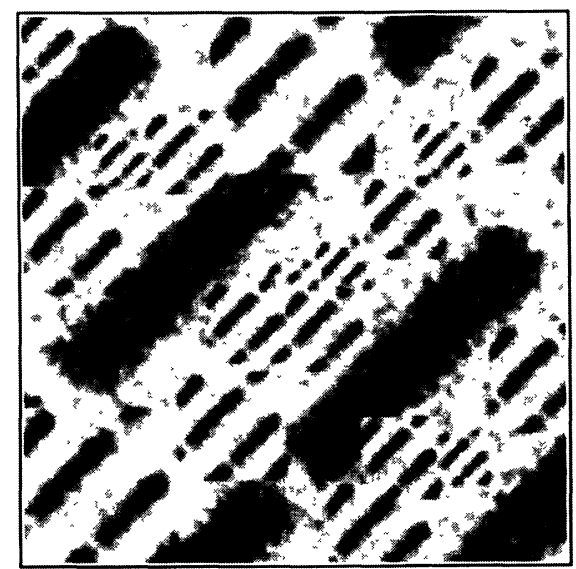

a)

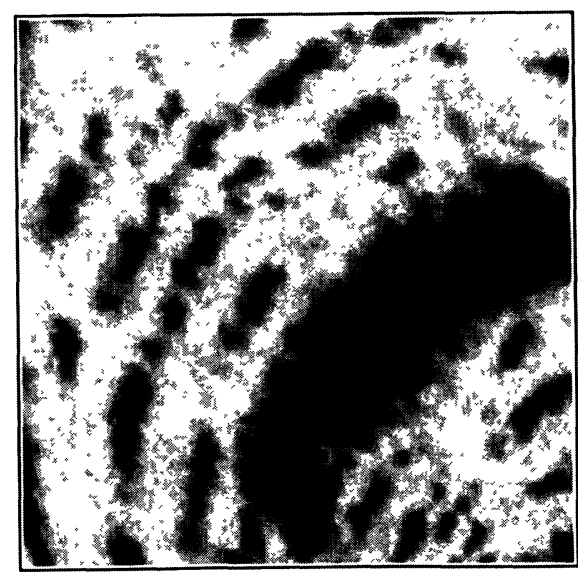

b)

Fig. 1. - a) Straight anisotropy test image, b) bent anisotropy test image, c) results of the application of grey-level granulometry on image (a) using six differently oriented linear structuring elements. d) results of the application of grey-level granulometry on image (a) using $45^{\circ}$ and $135^{\circ}$ clockwise linear structuring elements. Note the peak at 10 iterations for the $45^{\circ}$ clockwise curve and the peaks at 30 and 42 iterations for the $135^{\circ}$ clockwise curve. e) results of the application of grey-level granulometry on image (b) using six differently oriented linear structuring elements (see text for more details). 

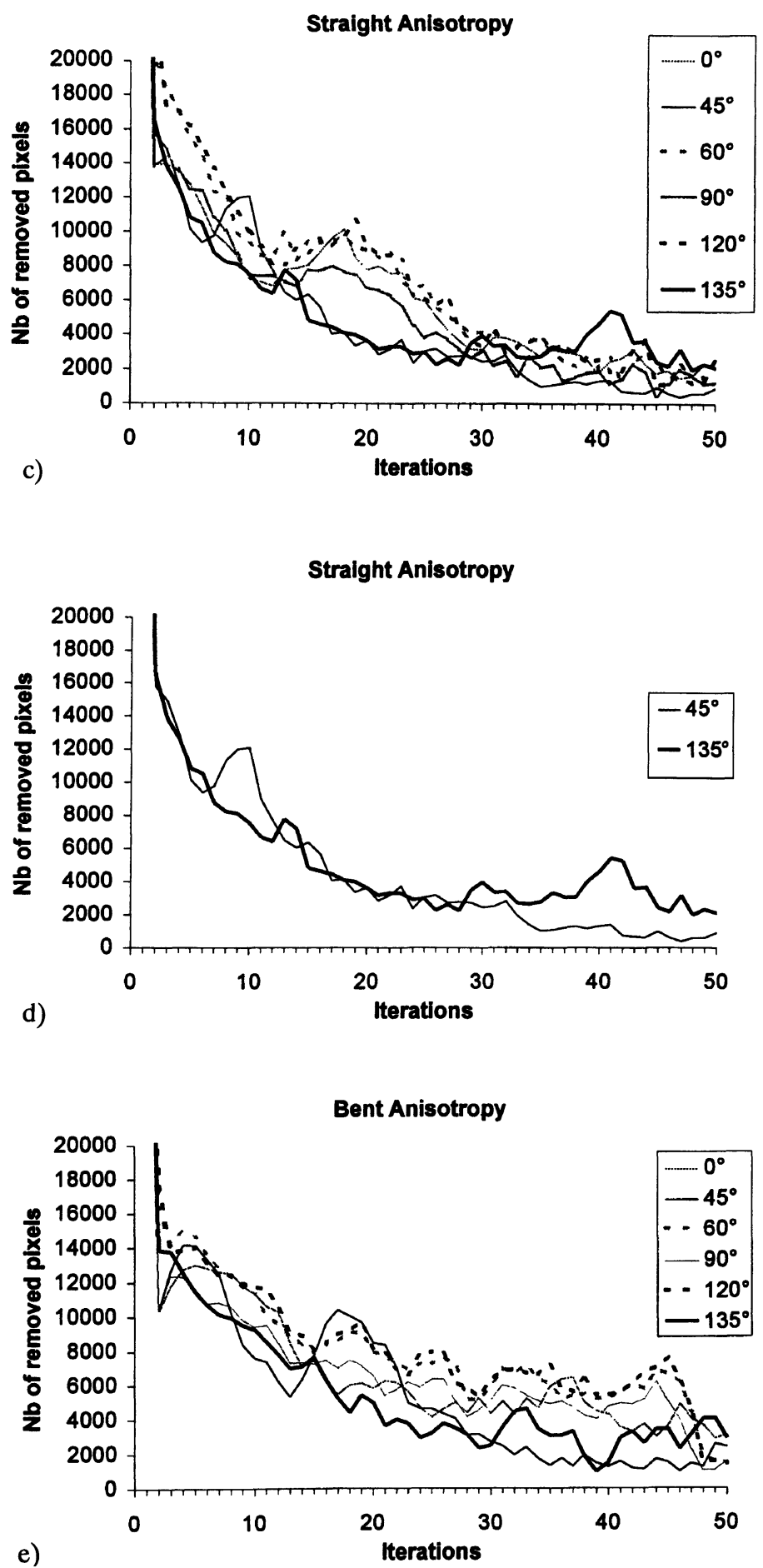

Fig. 1. - (continued) 


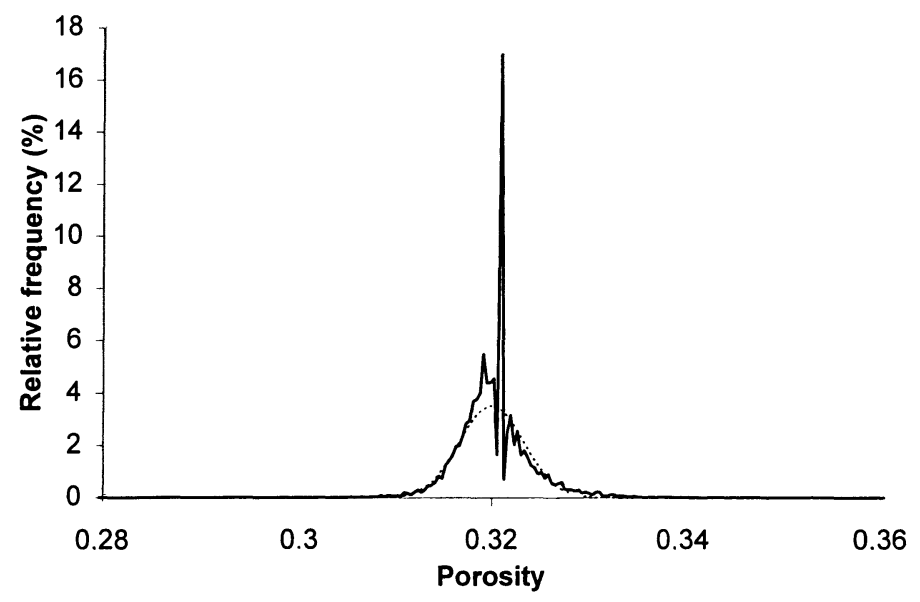

Fig. 2. - Grey-level histogram of the porosity image derived from the X-radiograph in Figure 7a. Note 1) the presence of a sharply defined peak corresponding to the sample average porosity and 2) the gap separating this peak from the higher porosity values. The Gaussian distribution fitted to this histogram is indicated in dotted line.

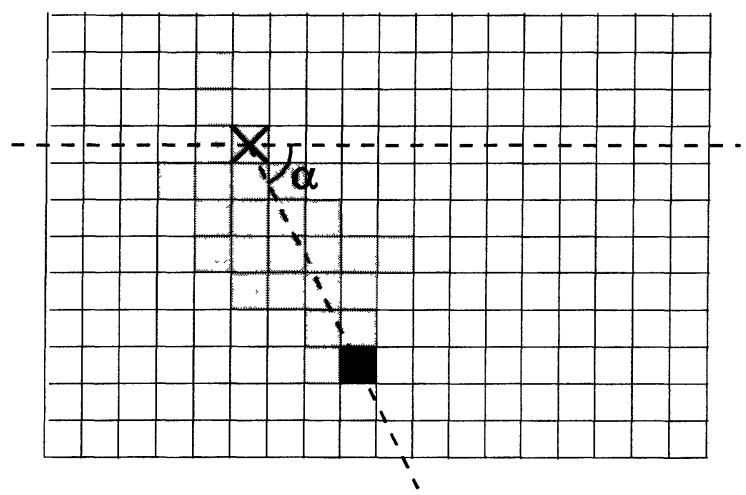

$\square$ Individual component

Х Current pixel

Current pixel's furthest neighbour

$\boldsymbol{\alpha}$ Clockwise angle to horizontal

Fig. 3. - Principle of the orientation labelling. For each individual component, the clockwise angle $\alpha$ between horizontal and the line linking each pixel to its furthest neighbour is computed and allocated to the corresponding pixel.

3.2.2 Orientation. - There is a number of parameters that can be used for orientation measurements on binary images [11]. However it is known that the most robust descriptors are those derived from all the pixels of the individual components rather than those taking into account only pixels around the periphery, for example, the maximum Feret's diameter [12]. 


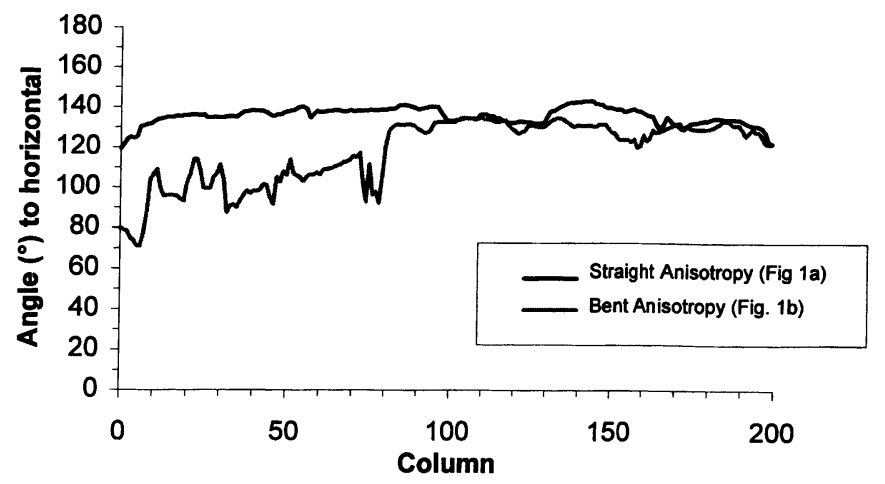

Fig. 4. - Plots of the column-wise orientations of pores patterns from test cases in Figures 1a and 1b.

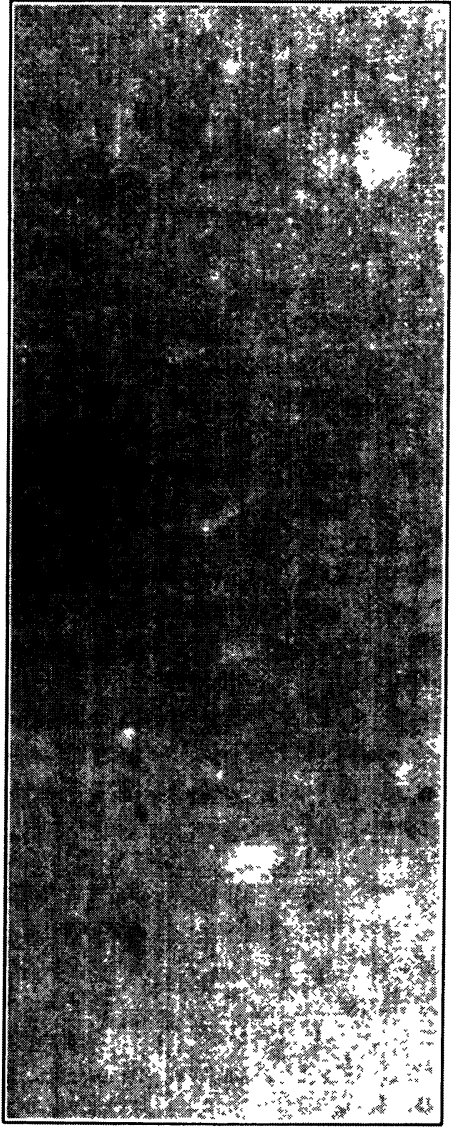

a)

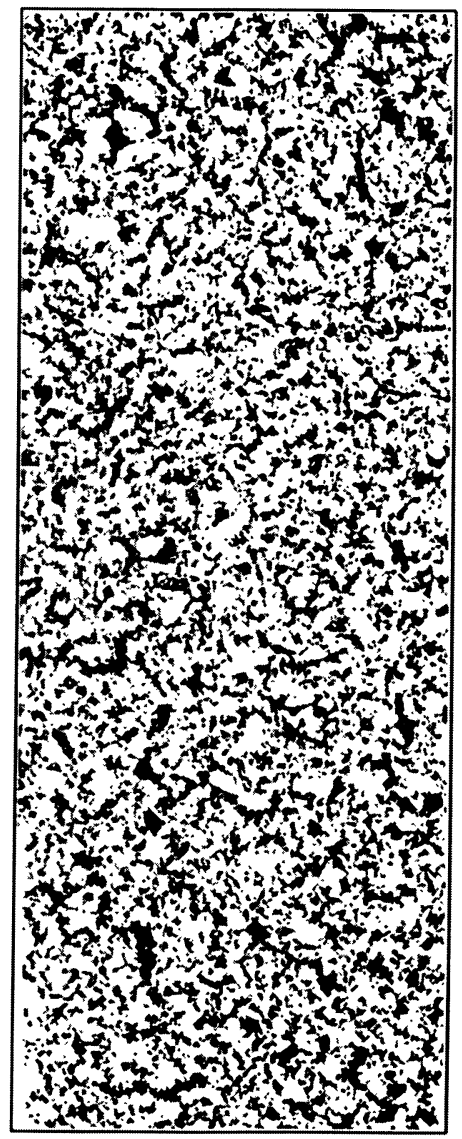

b)

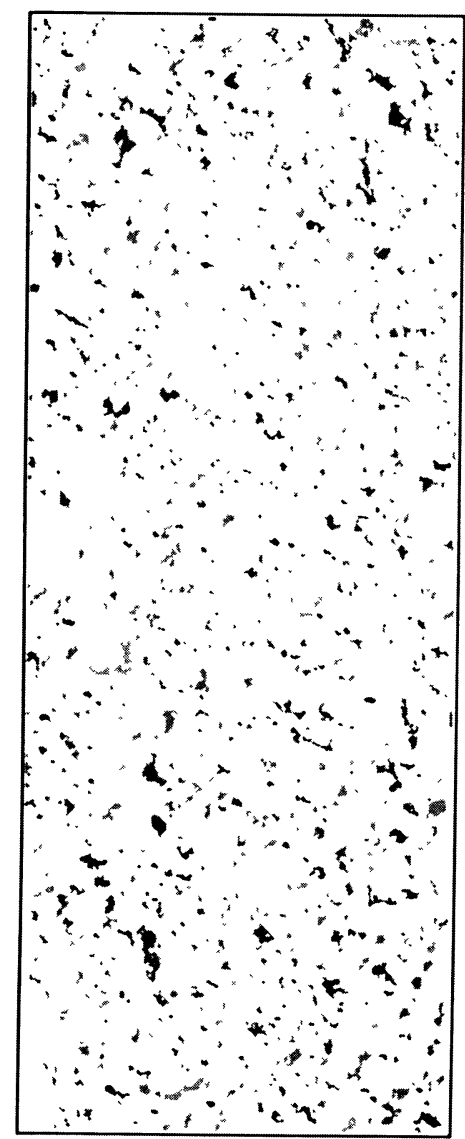

c)

Fig. 5. - Application of the binary method to a sample from Cameroon. a) Original X-radiograph (pores appear in black), b) binary image of pore related objects, c) orientation image (black $=0^{\circ}$ angle clockwise to horizontal, white $=180^{\circ}$ angle clockwise to horizontal). Actual size of images is $41 \times 106 \mathrm{~mm}$. 

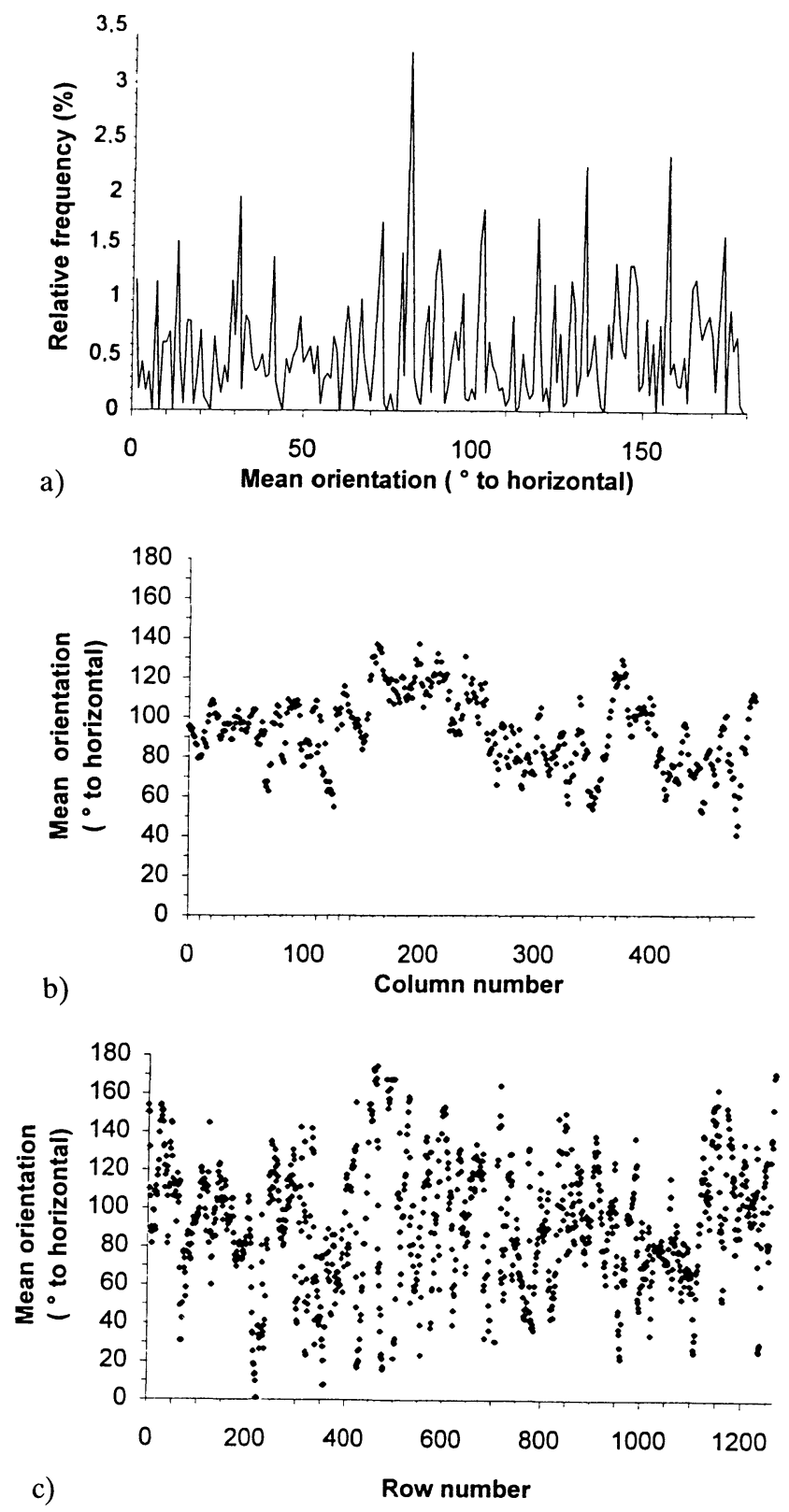

Fig. 6. - a) Histogram of the orientation image of Figure 5c. b) Plot of the column-wise orientations of pores. c) Plot of the row-wise orientations of pores. For this sample, the pore pattern do no exhibit any anisotropy.

Given that we are particularly interested in the problem of bent anisotropy, measurement of orientation of pores in the binary image was achieved by examining each pore and its position columnand row-wise in the image. First, each pore is given a unique label. Each pixel in each pore is marked with the direction of the pixel farthest from it within the pore (Fig. 3). This information is summarised by storing the mean and variance of each pore. 


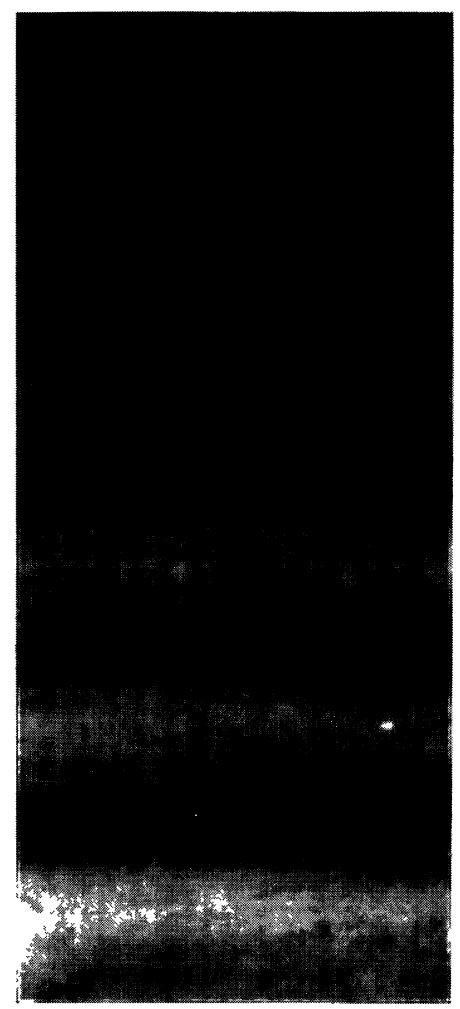

a)

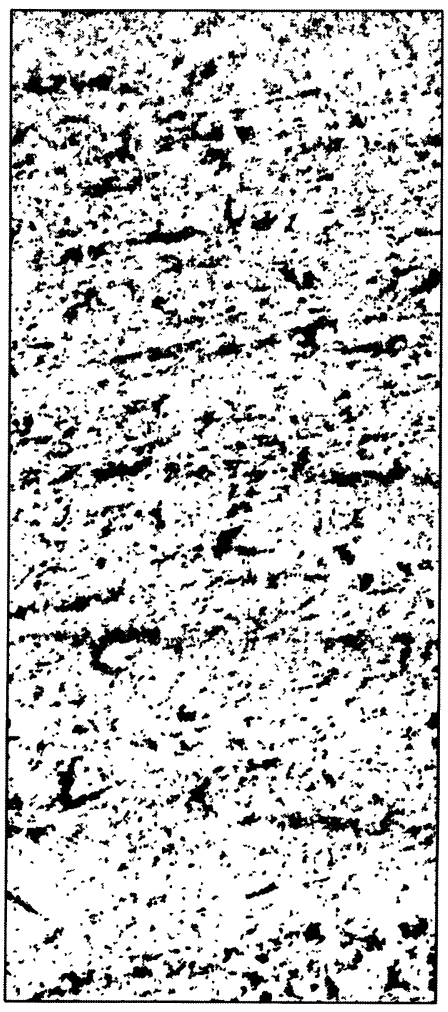

b)

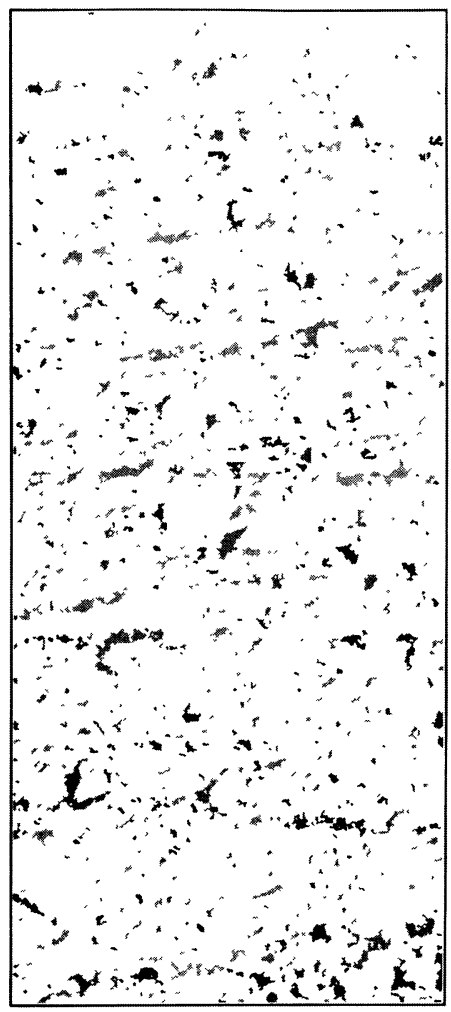

c)

Fig. 7. - Application of the binary method to a sample from India. a) Original X-radiograph (pores appear in black), b) binary image of pore related objects, c) orientation image with (black $=0^{\circ}$ angle clockwise to horizontal, white $=180^{\circ}$ angle clockwise to horizontal). Actual size of images is $42.5 \times 97.5 \mathrm{~mm}$.

In order to limit noise related to orientation measurement of very small components, five openings are arbitrary applied at the start of the orientation process. The result is a relatively disconnected and low noise binary image. Only components with sufficient elongation (with measured orientations ranging over less than $30^{\circ}$ ) are kept to form the final orientation image and the associated statistic files. It is noticeable that this process removes not only circular pores but also some low convexity components, e.g. star shaped pores.

3.2.3 Graphic Representation. - In addition to the final orientation images with individual components labelled with their mean orientation, it is informative to present a graphical summary of the orientation measurements. First, a frequency histogram of the orientation images can be plotted. This allows a direct assessment of the global degree of anisotropy of the pore pattern. However, such information loses the spatial context. Mean orientation as a function of height and width are also presented. Data used for these plots are the mean orientation computed for each column (or row).

As an illustration of this method, the plots presented in Figure 4 show the results obtained when it is applied to the same test images used to evaluate the grey-level granulometry approach (Figs. 1a, 1b). The binary measurements appear to describe the anisotropy and are easy to interpret despite 

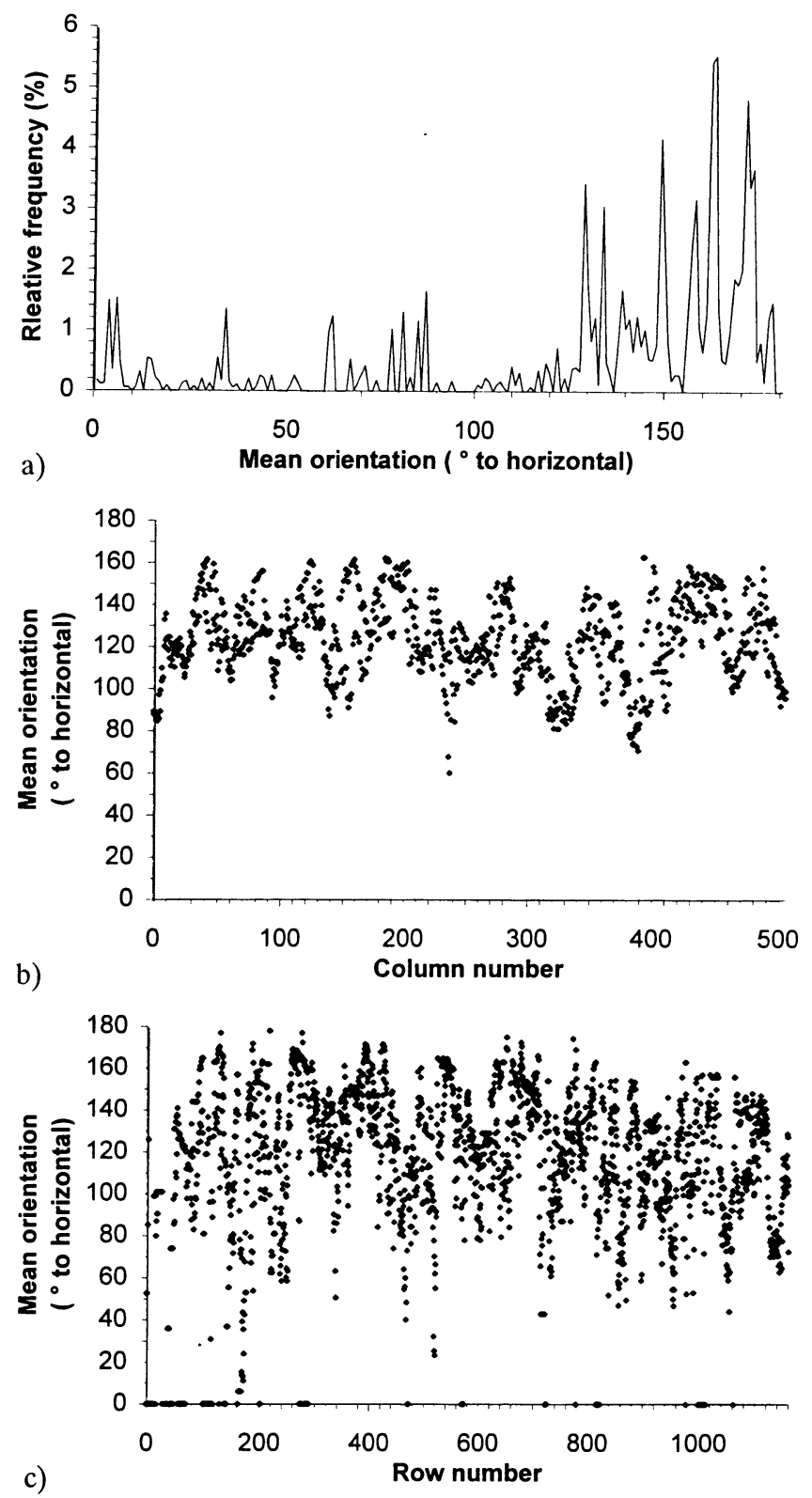

Fig. 8. - a) Histogram of the orientation image of Figure 7c. b) Plot of the column-wise orientations of pores. c) Plot of the row-wise orientations of pores. For this sample, the pore pattern exhibits a clear anisotropy slanted at approximately $160-170^{\circ}$ clockwise to horizontal.

the curvature of the pore pattern inspected. The measured orientations (clockwise) for the bent anisotropy gradually increase from the left to the right of the image. This is a result consistent with what can be seen on the image (despite a perturbation induced by the largest porous component) expressed as a rough stabilization of the plot between $120-140^{\circ}$. 


\section{Results}

The binary method is illustrated using two samples collected among the production of traditional potters in India and Cameroon. The results from the binary method will be only presented, because, as discussed above, it is more relevant to our particular problem and more informative and rapid than the fixed-grid method.

4.1 CAmeroon. - The sample from Cameroon was collected in the village of Mijivin (Extreme North province) and is an example of fashioning by the so-called beating technique. This technique consists of flattening a mass of clay on a mould by applying pressure with slapping hands.

The succession of images from the raw X-radiographic image to the orientation image is shown in Figure 5. The histogram of the orientation image shows an even distribution of orientations values, and no global preferential orientation of the pore pattern can be inferred for this sample (Fig. $6 a)$. The plot of mean orientation as a function of width shows a rather regular distribution centered on $90^{\circ}$ to horizontal (Fig. 6b). However, the plot of mean orientation versus height reveals a non-clustered distribution of the mean orientation (Fig. 6c). Therefore, from the comparison of the three graphics, one can assess that the pore pattern of this sample is quite isotropic.

4.2 INDIA - - The sample from India is a thrown vessel collected in the Uttam Nagar district (New Delhi). The deformation rate applied for forming this vessel was radically different from the one involved for the previous sample: throwing involves the use of a potter's wheel as a power supply to force the clay between the potter's fingers.

The succession of images from the raw X-radiographic image to the orientation image is shown in Figure 7. The histogram of the orientation image shows a higher representation of orientations between 130 and $180^{\circ}$ than other orientations, with a dominant peak at about $165^{\circ}$ (Fig. $8 \mathrm{a}$ ). The plot of mean orientation as a function of width shows a distribution of values centered around $130^{\circ}$ with regularly spaced clusters between 150 and $160^{\circ}$ for the columns 0 to 200 and clusters at $130-140^{\circ}$ for the other columns (Fig. 8b). The plot of mean orientation as a function of height shows a mean orientation distribution shifted to the $140-160^{\circ}$ orientations (Fig. 8c). This provides evidence of well-expressed anisotropy of the pore pattern of this sample.

\section{Conclusion}

A method based on combination of conventional X-radiography and image processing for quantification of orientation of pore patterns of deformed clay has been presented. The samples studied are ceramics for which the proposed approach provide calibrated data on the basis of which technological conclusions can be inferred. By comparison with conventional qualitative analysis, this provides progress for reproductibility and sensitivity of the diagnostics. Moreover, it is noticeable that our methodology is easy to perform, low-cost and relatively rapid, so that it can be considered well fitted to the field of archaeological research for which it has been designed.

\section{Acknowledgements}

The authors thank Dr V. Roux, CNRS, ERA 28, Meudon (France) and Dr. M. Delneuf, ORSTOM, Bondy (France) for providing experimental materials studied in this paper. Many thanks to Pr L.-M. Bresson, INAP-G, Grignon (France) for having paid kind attention to the development of this work and for illuminating advising. A.P. would like to express his gratitude to Dr A. Kretzschmar (INRA Zoologie Avignon), and to the staff at the INRA Zoologie Avignon, for support and critical discussions. 


\section{References}

[1] Rye O.S., Archaeometry 19 (1977) 205-211.

[2] Rye O.S., Pottery Technology. Principles and Reconstruction (Washington D. C., Taraxacum Press, 1981).

[3] Vandiver P.B., Sequential Slab Construction: A Near Eastern Pottery Production Technology, 80003000 B. C. Ph.D. Thesis, M.I.T, Cambridge (1985).

[4] Vandiver P.B., Paleorient 13 (1987) 9-36.

[5] Mc Govern P.E., Ancient ceramic technology and stylistic change: contrasting studies from Southwest and Southeast Asia. In: "J. Scientific Analysis in Archaeology", Henderson Ed. (Oxford University Committee for Archaeology) Monograph No. 19 / UCLA Institute of Archaelogy, Archaeol. Res. Tools 5 (1989) 63-81.

[6] Carr C., J. Archaeol. Sci. 17 (1990) 13-34.

[7] Blackmann M.J., Stein G.J. and Vandiver P.B., Amer. Antiquity 58 (1993) 60-80.

[8] Pierret A., Moran C.J. and Bresson L.-M., J. Archaeol. Sci. 23 (1996) 419-428.

[9] Carr C. and Riddick E.B. Jr., J. Archaeol. Sci. 17 (1990) 35-66.

[10] Serra J., Image Analysis and Mathematical Morphology, Vol I (London, Academic Press, 1982).

[11] Moran C.J., Image Processing and Micromorphology, in "Soils Micromorphology: Studies in Management and Genesis", A.J. Ringrose-Voase and G. Humphries, Eds., Developments in Soil Science 22 (Elsevier, 1994) 459-482.

[12] Russ J.C., The Image Processing Handbook, 2nd ed. (CRC Press, 1995). 